\title{
Conical Nanoparticles for Blood Disease Detection
}

\author{
Luigi La Spada, Renato Iovine, Richard Tarparelli, Lucio Vegni \\ Department of Engineering, Roma Tre University, Rome, Italy \\ Email: luigi.laspada@uniroma3.it,renato.iovine@uniroma3.it, richard.tarparelli@uniroma3.it,vegni@uniroma3.it
}

Received May 6, 2013; revised June 6, 2013; accepted June 14, 2013

Copyright (C) 2013 Luigi La Spada et al. This is an open access article distributed under the Creative Commons Attribution License, which permits unrestricted use, distribution, and reproduction in any medium, provided the original work is properly cited.

\begin{abstract}
Metallic nanoparticles play an important role in the design of sensing platforms. In this paper, a new electromagnetic study for conical metal nanoparticles, working in the Near Infrared and Visible frequency regime, is proposed. The structures consist of inclusions, arranged in an array configuration, embedded in a dielectric environment. The aim of this work is to develop new analytical models, in order to describe the nanoparticles electromagnetic behavior in terms of extinction cross-section (absorption and scattering). The closed-form formulas link the conical nanoparticles geometrical and electromagnetic parameters to their resonant frequency properties in terms of wavelength position, magnitude and bandwidth. The proposed models are compared to the numerical results and to the experimental ones, reported in literature. Good agreement is obtained. The proposed analytical formulas represent useful tools for sensing applications. For this reason, exploiting such models a new sensing platform able to detect different blood diseases is obtained. Numerical results confirm the capability of the proposed structure to be used as a sensing platform for medical diagnostics.
\end{abstract}

Keywords: Conical Nanoparticles; Analytical Models; Sensitivity; Sensing Platform; Blood Diseases

\section{Introduction}

Nanoparticles are of great scientific interest due to their potential role in several application fields such as medicine, optics and electronics. When an electromagnetic field interacts with metal particle, collective electron charge oscillations arise. Such a phenomenon is called Localized Surface Plasmon Resonance (LSPR). At the resonant condition nanoparticles exhibit enhanced electromagnetic near-field, highly localized in their neighborhood.

Moreover, far-field particle scattering is also enhanced by the resonance. Light intensity enhancement is a crucial aspect of LSPR structures and localization leads to a very high spatial resolution. Such particular optical properties of metallic nanoparticles make them suitable for several applications involving optical and photonic ones $[1,2]$, biochemical sensing and detection $[3,4]$, protein analysis [5,6], cell membrane function [7], biomedical applications $[8,9]$, food quality analysis [10] and imaging $[11,12]$. The use of nanoparticles in medicine offers some exciting applications, such as drugs delivery, heating therapy techniques, in vivo tumor cell targeting and early diagnostic techniques [13].

Past researches on metallic nanoparticles have concentrated only on two main issues: fabrication processes and experimental characterization techniques. The literature is almost silent on metal nanoparticles electromagnetic properties modeling. Nanoparticles analytical models turn out to be a useful tool in order to study their optical properties; in particular how such structures influence and interact with the surrounding environment, and how to manipulate, control and design their electromagnetic properties for specific required applications. Therefore, this paper attempts to present new design methods for conical metallic nanoparticles with desired electromagnetic properties. The findings of this study are expected to provide a greater understanding of their electromagnetic behavior and the possibility to use them for sensing applications. The choice of conical particles is up to their use for biomedical applications [14] and Near-Field Optical Microscopy Probes [15]. In addition to this, the tip ensures stronger electric field localization [16].

The article is structured as follows: first of all the electromagnetic properties of metallic conical nanoparticles are evaluated in terms of extinction cross section (absorption and scattering). For this purpose, new analytical closed-form formulas, linking the geometrical and the electromagnetic parameters of such nanoparticles with their resonant frequency properties, are evaluated. This is followed by a comparison among analytical, numerical and experimental results. The capability to manipulate and control the electromagnetic phenomenon, by exploiting the proposed analytical models, on the nanometer 
scale, opens up several possible applications. For this reason, in the second part of this article the use of nanoparticles for sensing applications is presented. In particular, their sensitivity performances are analyzed and discussed. Finally, by exploiting the proposed electromagnetic models a new sensing platform for the detection of different blood diseases is proposed.

\section{Analytical Models and Electromagnetic Properties of Conical Nanoparticles}

In this section the mathematical modeling of conical metal nanoparticles electromagnetic properties is developed.

First of all, the electromagnetic problem is formulated. Then, the absorption and scattering cross-section models are obtained. Finally, in order to verify the proposed analytical models, a comparison with full-wave simulations and experimental values, existing in literature, is reported.

\subsection{Formulation of the Electromagnetic Problem}

The resonant behavior of the individual structure is studied in terms of a quasi-static approximation. In particular, the general analytical expression of the nanoparticle polarizability is derived and the dependence of the inclusion polarizability on its geometry, its metallic electromagnetic properties, and the permittivity of the surrounding dielectric environment, is presented. The proposed structures consist of resonant metallic conical inclusions, arranged in an array configuration, whose frequency response is modified through the refractive index variation of the surrounding dielectric environment. Let us assume that the structure is excited by an impinging electromagnetic plane wave, having the electric field $\mathbf{E}$ parallel and the propagation vector $\mathbf{k}$ perpendicular to the nanoparticle axis, respectively (Figure 1). The geometrical parameters considered in this study are shown in Figure 1, being $a$ the bottom radius and $h$ the height of cone. The nanoparticles electromagnetic properties are revealed in terms of extinction cross-section. The nanostructure holds specific resonant frequency properties in terms of position, magnitude and bandwidth, depending on its geometrical (shape, dimensions) and on the electromagnetic

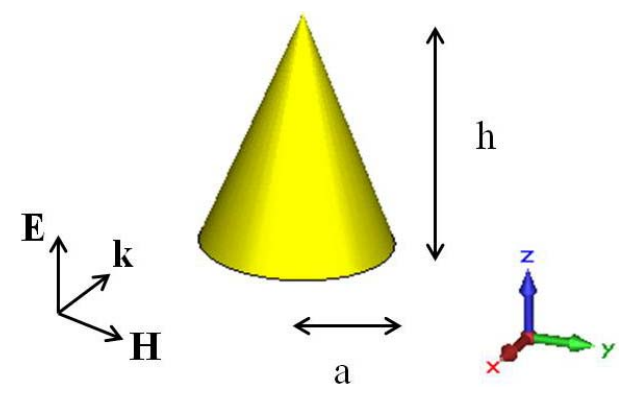

Figure 1. Conical nanoparticle geometry: $a$ bottom radius, $h$ height of cone. characteristics of the metal it is made of. In order to study the nanoparticle electromagnetic properties the following assumption must be done:

- the particle is considered "electrically small": its size is much smaller than the operative wavelength in the surrounding medium [17]. Consequently, its resonant behavior can be studied in terms of quasi-static approximation;

- the particle is considered homogeneous and the surrounding dielectric environment is a homogeneous, isotropic and non-absorbing medium.

\subsection{Absorption and Scattering Cross-Section Analytical Models}

Under such conditions the nanoparticles resonant optical properties can be evaluated starting from their polarizability expressions. It is well known that [18] the polarizability component along the $\mathrm{z}$-axis direction (in the limit of quasi-static approximation) can be expressed as:

$$
\alpha_{z}=V \varepsilon_{e} \frac{\varepsilon_{i}-\varepsilon_{e}}{\varepsilon_{e}+L_{z}\left(\varepsilon_{i}-\varepsilon_{e}\right)}
$$

where $V$ is the particle volume, $\varepsilon_{e}$ is the surrounding dielectric environment permittivity, $\varepsilon_{i}$ is the inclusion dielectric permittivity and $L_{z}$ is the depolarization factor. Therefore, as shown in (1) the inclusion electromagnetic properties are highly dependent on its geometrical parameters $(V$ and $L)$, on the metallic material properties $\left(\varepsilon_{i}\right)$ and on the surrounding environment $\left(\varepsilon_{e}\right)$.

Let us consider now that inclusions permittivity is described by a Drude model permittivity as follows:

$$
\varepsilon_{i}=\varepsilon_{\infty}-\frac{\omega_{p}^{2}}{\omega^{2}-j \omega \gamma}
$$

being $\varepsilon_{\infty}$ the permittivity at the high frequency limit, $\omega=$ $2 \pi f$ the angular frequency, $\omega_{p}$ the plasma frequency and $\gamma$ the collision frequency.

Such particles are embedded in a dispersionless and lossless surrounding environment with permittivity $\varepsilon_{e}$. It is well known that the dipolar polarizability $\alpha$ is maximized (the nanoparticle is at its resonant condition) when its denominator goes to zero in both its real and imaginary part [18]. By inserting (2) in the general particle polarizability expression (1) the resonant behavior of the nanoparticle is reached when:

$$
a_{4} f^{4}+a_{2} f^{2}+a_{0}=0
$$

where $f$ is the resonant frequency with:

$$
\begin{aligned}
& a_{4}=16 \pi^{4}\left[\left(L_{z}-1\right) \varepsilon_{e}-L_{z} \varepsilon_{\infty}\right]^{2} \\
& a_{2}=\gamma^{2} \frac{a_{4}}{4 \pi^{2}}+8 \pi^{2} \sqrt{a_{0}} \quad a_{0}=L_{z}^{2} \omega^{4}
\end{aligned}
$$

We obtain the corresponding resonant wavelength con- 
dition:

$$
\lambda=c \frac{\sqrt{\frac{\sqrt{a_{2}^{2}-4 a_{0} a_{4}-a_{2}}}{a_{0}}}}{\sqrt{2}}
$$

Using (5) it is possible to predict how the particle geometrical parameters $\left(L_{z}(a, h)\right)$, the metal electromagnetic properties $\left(\varepsilon_{\infty}\right.$ and $\gamma$ ) and the surrounding dielectric environment $\left(\varepsilon_{e}\right)$ affect its resonant behavior in terms of resonant position. To describe the entire nanoparticles electromagnetic behavior it is necessary to evaluate their electromagnetic extinction cross section properties, in terms of scattering and absorption. Separated evaluation of the absorption and scattering phenomenon for each nanoparticle is crucial to understand why certain structures are preferred to others for specific applications. In other words, by evaluating closed-form formulas for extinction cross-section properties, it is possible to correlate the particle electromagnetic properties with its geometrical characteristics, in order to describe its resonant behavior, in terms of wavelength position, magnitude and bandwidth. The analytical electromagnetic solution exists only for a restricted number of particle geometries, such as sphere and cylinder [19], cube [20], disc and needle $[21,22]$. For any other arbitrary shapes the electromagnetic solution is given by numerical approaches [23].

It's known [24] that the corresponding expressions, linking the inclusion polarizability to its extinction properties, for absorption cross-section $\left(C_{a b s}\right)$ and scattering cross-section $\left(C_{s c a}\right)$ read respectively:

$$
\begin{gathered}
C_{a b s}=k \operatorname{Im}[\alpha] \\
C_{s c a}=\frac{k^{4}}{6 \pi}|\alpha|^{2}
\end{gathered}
$$

where $k=2 \pi n / \lambda$ is the wave-number, $\lambda$ is the wavelength and $n=\sqrt{ }_{\varepsilon}$ is the refractive index of the surrounding dielectric environment.

Then, by following the same procedure in [24] and considering the electric field polarization and the particle geometry, the absorption and scattering cross-section analytical models have been developed and here reported, respectively:

$$
\begin{gathered}
C_{a b s}=k \frac{\pi h a^{2}}{3} \operatorname{Im}\left[\frac{\varepsilon_{e}\left(\varepsilon_{i}-\varepsilon_{e}\right)}{\varepsilon_{e}+L_{\text {cone }}\left(\varepsilon_{i}-\varepsilon_{e}\right)}\right] \\
C_{\text {sca }}=\frac{k^{4}}{6 \pi}\left|\frac{\left(\frac{\pi h a^{2}}{3}\right) \varepsilon_{e}\left(\varepsilon_{i}-\varepsilon_{e}\right)}{\varepsilon_{e}+L_{\text {cone }}\left(\varepsilon_{i}-\varepsilon_{e}\right)}\right|
\end{gathered}
$$

where $L_{\text {cone }}$ reads:

$$
L_{\text {cone }}=\frac{1}{2}\left(1-\frac{1}{\sqrt{4 \frac{a^{2}}{h^{2}}+1}}\right)
$$

In Figure 2 a comparison between analytical and numerical results [25], for extinction cross-section spectra, is presented. In particular:

- for gold nanoparticles, experimental values [26] of the complex permittivity function have been used;

- the surrounding dielectric medium is considered to be vacuum.

\subsection{Comparison between Analytical Model, Numerical Simulations and Experimental Values}

The proposed analytical models are verified through the comparison with full-wave numerical simulations, and the experimental data existing in literature for gold [15], silver [27] and copper [28] conical particles, for different geometrical parameter values as shown in Table 1, where the relative errors are evaluated and reported. Good agreement among all three results was reached. The relative errors are expressed as:

$$
\begin{aligned}
& E_{a-n}=\frac{\lambda_{\text {analitical }}-\lambda_{\text {numerical }}}{\lambda_{\text {numerical }}} \\
& E_{a-e}=\frac{\lambda_{\text {analitical }}-\lambda_{\text {experimental }}}{\lambda_{\text {experimental }}}
\end{aligned}
$$

\section{Sensitivity Analysis}

In this paragraph the sensitivity properties of conical nanoparticles are evaluated. Sensitivity is commonly de-

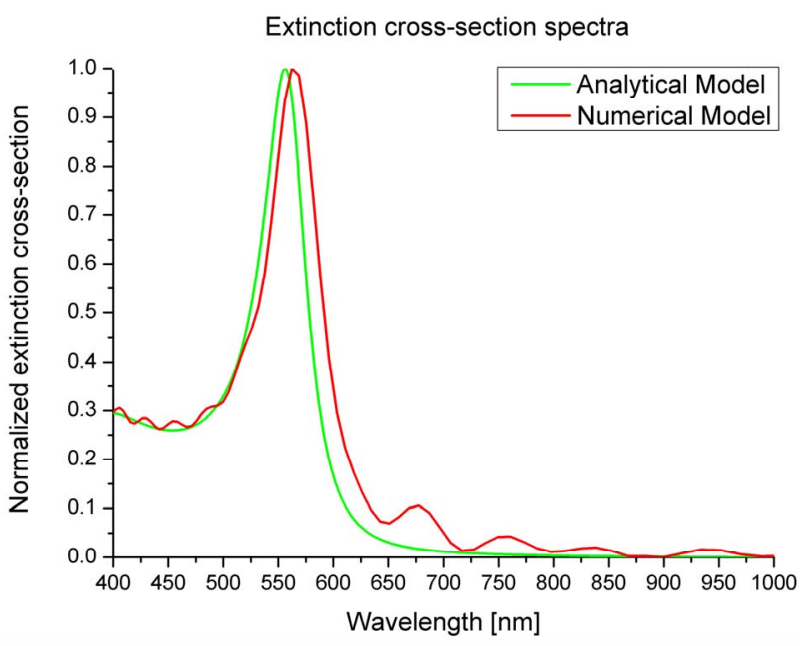

Figure 2. Comparison between numerical and analytical model for extinction cross-section $(a=20 \mathrm{~nm} ; \boldsymbol{h}=20 \mathrm{~nm})$. 
Table 1. Comparison of resonant wavelengths for gold, silver and copper conical particles: analytical, numerical and experimental values (gold [15], silver [27] and copper [28]).

\begin{tabular}{|c|c|c|c|c|c|c|}
\hline \multirow{2}{*}{ Particle } & \multirow{2}{*}{ Size $[\mathrm{nm}]$} & \multicolumn{3}{|c|}{ Resonant wavelength $(\lambda[\mathrm{nm}])$} & \multirow{2}{*}{$E_{a-n}(\%)$} & \multirow{2}{*}{$E_{a-e}(\%$} \\
\hline & & Analytical values & Numerical values & Experimental values & & \\
\hline \multirow{3}{*}{ gold } & $a=45, h=25$ & 540 & 570 & 550 & 5.263 & 1.818 \\
\hline & $a=45, h=50$ & 560 & 590 & 570 & 5.085 & 1.754 \\
\hline & $a=45, h=100$ & 620 & 650 & 610 & 4.615 & 1.639 \\
\hline \multirow{3}{*}{ silver } & $a=28, h=93$ & 650 & 670 & 650 & 2.985 & 0 \\
\hline & $a=46, h=100$ & 580 & 600 & 586 & 3.333 & 1.024 \\
\hline & $a=70, h=115$ & 582 & 610 & 588 & 4.590 & 1.020 \\
\hline \multirow{3}{*}{ copper } & $a=190, h=20$ & 750 & 780 & 764 & 3.850 & 1.83 \\
\hline & $a=190, h=40$ & 720 & 740 & 713 & 2.70 & 0.98 \\
\hline & $a=190, h=60$ & 660 & 700 & 685 & 5.71 & 3.65 \\
\hline
\end{tabular}

fined as $S=\Delta \lambda / \Delta n$, expressed in $n m / R I U$ (Refractive Index Unit). Typically, if the refractive index variation range is sufficiently narrow, both analytical results (Figure 3(a)) and full-wave simulations (Figure 3(b)) highlight that the input-output relation between refractive index and resonant wavelength position can be considered linear. A test material, surrounding the nanoparticle, with a varying refractive index $n$ in the range $1-3$ has been chosen.

In Table 2, instead, analytical, numerical and experimental sensitivity for gold [29], silver [30] and copper [31] conical particles are compared.

\section{The Nanocone-Based Sensor}

In this section the design of a gold conical nanoparticle array is proposed. As it will be shown by numerical results, the proposed structure can be used as a sensing platform for the detection of blood diseases.

Dielectric properties of biological samples can be described by the complex refractive index as:

$$
n_{c}=n_{r}+j k
$$

where $n_{r}$ is the real part and $k$ is the imaginary part of refractive index of the sample. Its electromagnetic properties are related to the real and imaginary part of the refractive index as a function of the frequency. Tissue dielectric properties and their frequency response are the results of the interaction between the electromagnetic radiation and their constituents at molecular and cellular level. These variations imply significant changes in their electromagnetic properties.

It's well known that hematological diseases induce structural, biochemical and mechanical changes in Red Blood Cells (RBCs) [32]. The structural variations imply significant changes in cell electromagnetic properties.

The refractive indices of different kind of RBCs, in the optical frequency range, differ in their real and imaginary

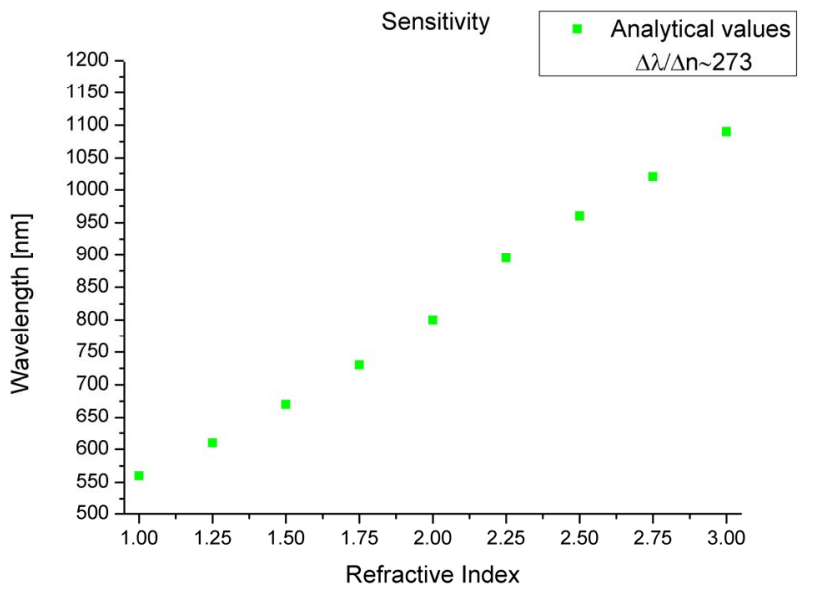

(a)

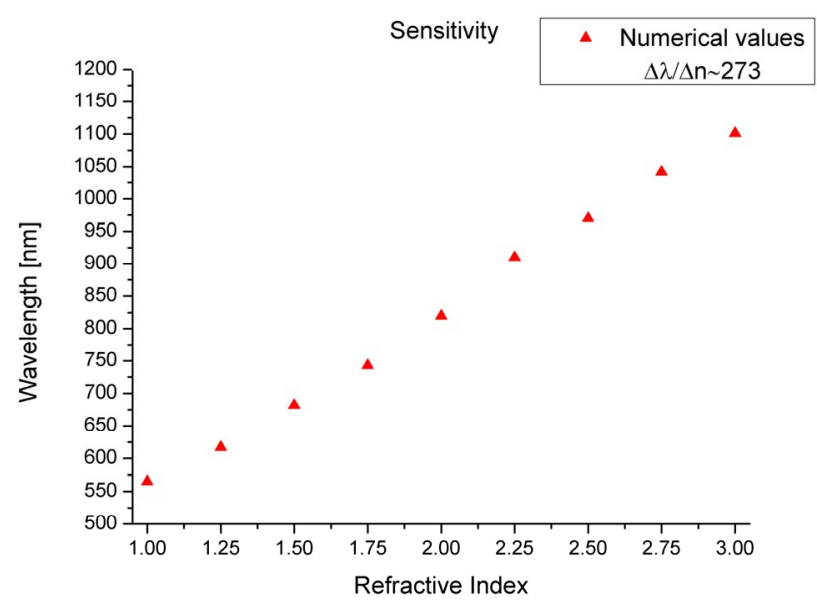

(b)

Figure 3. Variation of the resonant wavelength as a function of the refractive index of the surrounding medium $(a=20$ $\mathrm{nm} ; \boldsymbol{h}=\mathbf{2 0} \mathrm{nm}$, gold nanoparticle): (a) analytical values; (b) full-wave simulations results.

part. As a result it is possible to detect such differences by permittivity measurements on the considered sample. 
Table 2. Sensitivity values for gold, silver and copper conical particles: comparison among analytical, numerical and experimental values (gold [29], silver [30] and copper [31]).

\begin{tabular}{cccc}
\hline \multirow{2}{*}{ Particle } & \multicolumn{3}{c}{ Sensitivity [nm/RIU] } \\
\cline { 2 - 4 } & $\begin{array}{c}\text { Analytical } \\
\text { sensitivity }\end{array}$ & $\begin{array}{c}\text { Numerical } \\
\text { sensitivity }\end{array}$ & $\begin{array}{c}\text { Experimental } \\
\text { sensitivity }\end{array}$ \\
\hline gold & 300 & 275 & 239 \\
silver & 200 & 210 & 197 \\
copper & 280 & 250 & 200 \\
\hline
\end{tabular}

Therefore the main purpose of this paragraph is to correlate the sensor electromagnetic properties with the ones of the substance under test.

To detect the aforementioned changes (corresponding to different blood pathological states) a new sensing platform is proposed. In Figure $\mathbf{4}$ the sensing system operation pattern is shown. The sensor, without any Material Under Test (MUT), has a specific resonant frequency. Once the material to study is placed, the system "sensorMUT" is illuminated by an electromagnetic field. The nanoparticle-based sensor (yellow) is in direct contact with the biological sample (green). In this configuration, the MUT electromagnetic properties play a crucial role in the variation of the total effective permittivity and of the sensing platform scattering properties. The signal detected (scattering cross-section) will have the resonant wavelength position, magnitude and bandwidth depending on the electromagnetic characteristics of the overall system "sensor-MUT". In particular, this sensor consists of gold conical nanoparticles arranged in an array configuration, deposited on a $\mathrm{SiO}_{2}$ substrate $\left(\varepsilon_{\mathrm{SiO}_{2}}=2.08\right)$ as shown in Figure 4.

The possibility to implement metallic cones on flat dielectric substrates is well established in literature, several kind of fabrication techniques are possible, for example: nano-imprint lithography and electron beam evaporation [33], electron beam induced deposition [34], templating approach [35] and nanotransfer printing (nTP) method [36].

By using the analytical models proposed in the previous section, a new sensing platform has been designed to resonate in the range $900-1200 \mathrm{~nm}$.

To describe the electromagnetic behavior of the biological sample under test, RBCs refractive index models proposed in [37] have been exploited. The scattering coefficients properties of the sensor change their position, depending on the different RBCs structural modifications (Figure 5). As a result, the sensor is capable to detect human red blood cells structural modifications, allowing us to detect different blood diseases, by refractive index measurements.

As shown in Figure 5 different spectral responses have been reported in order to show the sensor capability

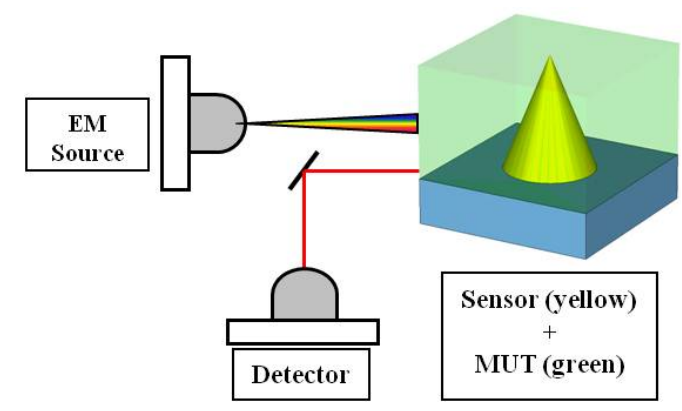

Figure 4. Sensing system operation pattern.

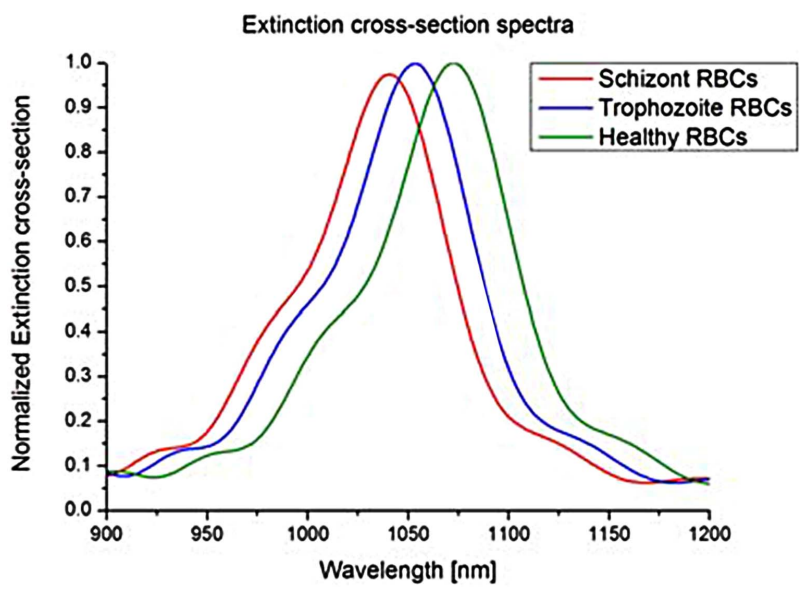

Figure 5. Scattering cross-section spectra for different structural changes in RBCs $(a=20 \mathrm{~nm} ; h=100 \mathrm{~nm})$.

to distinguish healthy RBCs from specific diseases such as Schizont and Trophozoite malaria.

\section{Conclusions}

In this paper a new study on metallic nanoparticles electromagnetic properties modeling was proposed. The considered structures were metallic cones, embedded in a surrounding dielectric environment, working in the Infrared and Optical frequency regime.

The electromagnetic modeling of nanoparticles was obtained in order to design new sensing platform. In this regard, new analytical models describing absorption and scattering cross-section properties, in terms of wavelength position, magnitude and amplitude width, were developed. Such new design formulas link the nanoparticles resonant properties with their structural and electromagnetic parameters. Then, the proposed models were compared to numerical simulation results and experimental data. A good agreement among analytical, numerical and experimental values was obtained. The sensitivity of the nanoparticles was studied and discussed. The possibility to control nanoparticles electromagnetic properties, by exploiting the proposed analytical models, paves the way to several possible applications. In particular, the proosed structure was designed in order to detect different 
blood diseases. Numerical results have demonstrated the capability of conical particles array to be used as a sensing platform for medical diagnostics.

\section{REFERENCES}

[1] Y. F. Chau, Z.-H. Jiang, H. Y. Li, G. M. Lin, F. L. Wu and W. H. Lin, "Localized Resonance of Composite Core-Shell Nanospheres, Nanobars and Nanospherical Chains," Progress in Electromagnetics Research, Vol. 28, 2011, pp. 183-199. doi:10.2528/PIERB10102705

[2] J. B. Pendry, "Playing Tricks with Light," Science, Vol. 285, No. 5434, 1999, pp. 1687-1688. doi:10.1126/science.285.5434.1687

[3] A. El-Ansary and L.M. Faddah, "Nanoparticles as Biochemical Sensors," Nanotechnology, Science and Applications, Vol. 3, 2010, pp. 65-76. doi:10.2147/NSA.S8199

[4] X.-J. Chen, B. L. Sanchez-Gaytan, Z. Qian and S.-J. Park, "Noble Metal Nanoparticles in DNA Detection and Delivery," WIREs Nanomedicine and Nanobiotechnology, Vol. 4, No. 3, 2012, pp. 273-290. doi:10.1002/wnan.1159

[5] X. Yang, J. Li, H. Pei, D. Li, Y. Zhao, J. Gao, J. Lu, J. Shi, C. Fan and Q. Huang, "Pattern Recognition Analysis of Proteins Using DNA-Decorated Catalytic Gold Nanoparticles," 2013. doi:10.1002/smll.201202772

[6] C. Nietzold and F. Lisdat, "Fast Protein Detection Using Absorption Properties of Gold Nanoparticles," Analyst, Vol. 137, No. 12, 2012, pp. 2821-2826. doi:10.1039/C2AN35054H

[7] R. Bukasov, T. A. Ali, P. Nordlander and J. S. ShumakerParry, "Probing the Plasmonic Nearfield of Gold Nanocrescent Antennas," ACS Nano, Vol. 4, No. 11, 2010, pp. 6639-6650. doi:10.1021/nn101994t

[8] W. J. Galush, S. A. Shelby, M. J. Mulvihill, A. Tao, P. Yang and J. T. Groves, "A Nanocube Plasmonic Sensor for Molecular Binding on Membrane Surfaces," Nano Letters, Vol. 9, No. 5, 2009, pp. 2077-2082. doi: $10.1021 / \mathrm{n} 1900513 \mathrm{k}$

[9] N. L. Rosi and C. A. Mirkin, "Nanostructures in Biodiagnostics," Chemical Reviews, Vol. 105, No. 4, 2005, pp. 1547-1562. doi:10.1021/cr030067f

[10] H. M. Hiep, T. Endo, K. Kerman, M. Chikae, D. K. Kim, S. Yamamura, Y. Takamura and E. Tamiya, "A Localized Surface Plasmon Resonance Based Immunosensor for the Detection of Casein in Milk," Science and Technology of Advanced Materials, Vol. 8, No. 4, 2007, pp. 331-338. doi:10.1016/j.stam.2006.12.010

[11] D. Yelin, D. Oron, S. Thiberge, E. Moses, Y. Silberberg and I. Willner, "Multiphoton Plasmon-Resonance Microscopy," Optics Express, Vol. 11, No. 9, 2003, pp. 13851391. doi:10.1364/OE.11.001385

[12] K. Jakobsohn, M. Motiei, M. Sinvani and R. Popovtzer, "Towards Real-Time Detection of Tumor Margins Using Photothermal Imaging of Immune-Targeted Gold Nanoparticles," International Journal of Nanomedicine, Vol. 7, 2012, pp. 4707-4713. doi:10.2147/IJN.S34157

[13] W. Cai, T. Gao, H. Hong and J. Sun, "Applications of
Gold Nanoparticles in Cancer Nanotechnology," Nanotechnology, Science and Applications, Vol. 1, 2008, pp. 17-32. doi:10.2147/NSA.S3788

[14] N. A. Issa and R. Guckenberger, "Fluorescence near Metal Tips: The Roles of Energy Transfer and Surface Plasmon Polaritons," Optics Express, 2007, Vol. 15, No. 19, pp. 12131-12144. doi:10.1364/OE.15.012131

[15] M. Fleischer, A. Weber-Bargioni, M. V. P. Altoe, A. M. Schwartzberg, P. J. Schck, S. Cabrini and D. P. Kern, "Gold Nanocone Near-Field Scanning Optical Microscopy Probes," ACS Nano, Vol. 5, No. 4, 2011, pp. 2570-2579. doi:10.1021/nn102199u

[16] M. R. Gartia, M. Lu and G. L. Liu, "Surface Plasmon Coupled Whispering Gallery Mode for Guided and FreeSpace Electromagnetic Waves," Plasmonics, Vol. 8, No. 2, 2012, pp. 361-368. doi:10.1007/s11468-012-9398-5

[17] C. Bohren and D. Huffmann, "Absorption and Scattering of Light by Small Particles," John Wiley, New York, 1983.

[18] L. La Spada, R. Iovine and L. Vegni, "Nanoparticle Electromagnetic Properties for Sensing Applications," Advances in Nanoparticles, Vol. 1, No. 2, 2012, pp. 9-14. doi: 10.4236/anp.2012.12002

[19] A. D. Yaghjian, "Electric Dyadic Green's Functions in the Source Region," Proceedings of IEEE, Vol. 68, No. 2, 1980, pp. 248-263.

[20] J. Avelin, A. N. Arslan, J. Brännback, M. Flykt, C. Icheln, J. Juntunen, K. Kärkkäinen, T. Niemi, O. Nieminen, T. Tares, C. Toma, T. Uusitupa and A. Sihvola, "Electric Fields in the Source Region: The Depolarization Dyadic for a Cubic Cavity," Electrical Engineering, Vol. 81, No. 4, 1998, pp. 199-202. doi:10.1007/BF01233270

[21] L. D. Landau and E. M. Lifshitz, "Electrodynamics of Continuous Media," 2nd Edition, Pergamon Press, Oxford, 1984

[22] A. Sihvola, "Electromagnetic Mixing Formulas and Applications," The Institution of Engineering and Technology, London, 2008.

[23] A. Sihvola, "Dielectric Polarization and Particle Shape Effects," Journal of Nanomaterials, Vol. 2007, No. 1, 2007, pp. 1-9. doi:10.1155/2007/45090

[24] J. G. Van Bladel, "Electromagnetic Fields," John Wiley \& Sons, Hoboken, 2007.

[25] CST Computer Simulation Technology. www.cst.com

[26] P. B. Johnson and R. W. Christy, "Optical Constants of the Noble Metals," Physical Review B, Vol. 6, No. 12, 1972, pp. 4370-4379. doi:10.1103/PhysRevB.6.4370

[27] T. R. Jensen, M. L. Duval, K. L. Kelly, A. A. Lazarides, G. C. Schatz and R. P. Van Duyne, "Nanosphere Litography: Effect of the External Dielectric Medium on the Surface Plasmon Resonance Spectrum of a Periodic Array of Silver Nanoparticles," Journal of Physical Chemistry B, Vol. 103, No. 45, 1999, pp. 9846-9853. doi:10.1021/jp9926802

[28] H. C. George, Z. Jing, M. H. Erin, C. S. George and R. P. Van Duyne, "Plasmonic Properties of Copper Nanoparticles Fabricated by Nanosphere Lithography," Nano Letters, Vol. 7, No. 7, 2007, pp. 1947-1952. 
[29] P. Y. Chung, T. H. Lin, G. Schultz, C. Batich and P. Jiang, "Nanopyramid Surface Plasmon Resonance Sensors," Applied Physics Letters, Vol. 96, No. 26, 2010, Article ID: 2611081. doi:10.1063/1.3460273

[30] A. D. McFarl and R. P. Van Duyne, "Single Silver Nanoparticles as Real-Time Optical Sensors with Zeptomole Sensitivity," Nano Letters, Vol. 3, No. 8, 2003, pp. 10571062. doi:0.1021/n1034372s

[31] J. S. Sekhon and S S Verma, "Refractive Index Sensitivity Analysis of $\mathrm{Ag}, \mathrm{Au}$, and $\mathrm{Cu}$ Nanoparticles," Plasmonics, Vol. 6, No. 2, 2011, pp. 311-317. doi:10.1007/s11468-011-9206-7

[32] A. Kilejian, "Characterization of a Protein Correlated with the Production of Knob-Like Protrusions on Membranes of Erythrocytes Infected with Plasmodium Falcuparum," Proceedings of the National Academy of Sciences of the United State America, Vol. 76, No. 9, 1979, pp. 4650-4653. doi:10.1073/pnas.76.9.4650

[33] J. M. Kontio, J. Simonen, J. Tommila and M. Pessa, "Arrays of Metallic Nanocones Fabricated by UV-Nanoimprint Lithography," Microelectronic Engineering, Vol. 87, No. 9, 2010, pp. 1711-1715. doi:10.1016/j.mee.2009.08.015
[34] M. Fleischer, A. Weber-Bargioni, S. Cabrini and D. P. Kern, "Fabrication of Metallic Nanocones by Induced Deposition of Etch Masks and Ion Milling," Microelectronic Engineering, Vol. 88, No. 8, 2011, pp. 2247-2250. doi:10.1016/j.mee.2011.02.090

[35] D. Di, P. Dong, J. Chen, J. Chen, Z. Zhou, X. Wu and S. $\mathrm{Li}$, "Inexpensive and Fast Fabrication of Ordered Gold Nanocone Arrays," IEEE International Conference on Nano/Micro Engineered and Molecular Systems, Kaohsiung, 20-23 February 2011, pp. 555-558. doi:10.1109/NEMS.2011.6017416

[36] T. Kim, J. Kim, S. Jun Son and S. Seo, "Gold Nanocones Fabricated by Nanotransfer Printing and Their Application for Field Emission," Nanotechnology, Vol. 19, No. 39, 2008. doi:10.1088/0957-4484/19/29/295302

[37] Y. Park, M. Diez-Silva, G. Popescu, G. Lykotrafitis, W. Choi, M. S. Feld and S. Suresh, "Refractive Index Maps and Membrane Dynamics of Human Red Blood Cells Parasitized by Plasmodium Falciparum," Proceedings of the National Academy of Sciences of the United States of America, Vol. 105, No. 37, 2008, pp. 13730-13735. doi:10.1073/pnas.0806100105 